\title{
Novel software for medical and technical Thermal Object Identification (TOI) using dynamic temperature measurements by fast IR cameras
}

\author{
by M. Strakowska*, P. Chatzipanagiotou* ${ }^{* *}$ G. De Mey ${ }^{* * *}$, V. Chatziathanasiou* ${ }^{* *}$ B. Więcek \\ * Lodz University of Technology, Institute of Electronics, 211/215 Wólczańska St. 90-924 Łódź, Poland, \\ maria.strakowska@p.lodz.pl \\ ** Aristotle University Of Thessaloniki, Department Of Electrical And Computer Engineering, 54124 Thessaloniki, \\ Greece \\ ${ }^{* * *}$ Gent University, Department of Electronics and Information Systems, Sint-Pietersnieuwstraat 41, B-9000 Gent, \\ Belgium
}

\begin{abstract}
This paper presents a thermal analysis and methodology of thermal parameters identification of thermal multilayer structures using thermographic measurements after dynamic heating (or cooling) the investigated objects. During the thermal transient process, thermal images are recorded, with the highest possible frame rate of the camera. Then, temperature and power signals are transformed into the frequency domain, to get finally the thermal time constants distribution corresponding to the thermal parameters of the structure. A brief description of TOI program developed in MATLAB environment as well as the exemplary identifications of 2 different thermal objects are shown.
\end{abstract}

\section{Introduction}

Many scientific efforts have been recently undertaken for dynamic object identification in different applications [1-26]. In heat transfer domain, there is the commercially available software for thermal object characterisation based on Network Identification by Deconvolution (NID) technique [2,3]. It is mainly dedicated for thermal characterization of microelectronic devices and systems [25]. It is limited to the contact temperature measurement using either internally built-in or external contact temperature sensors. For IR temperature measurements, no tools are available for thermal object identification. In addition, the NID methodology is not directly suitable for medical applications, e.g., for screening of skin pathologies and inflammations [22]

In this paper, we present a simple software tool for dynamic thermal object identification written in MATLAB environment. The block diagram of the overall algorithm of Thermal Object Identification (TOI) is presented in fig. 1. Firstly, the temperature vs. time is measured on the surface of the object using a thermographic camera. It is recommended to use the fast thermal camera with the frame rate of a few hundreds $\mathrm{Hz}$ at least. Then, the preprocessing is performed for noise reduction. Next, the Laplace transform of both the temperature and power is numerically calculated in order to get the thermal impedance (transfer function). The thermal impedance is approximated by the ratio of polynomials with the declared order. Typically for a thermal object, the order of numerator is one less than the order of denominator. Using the optimization one can get the coefficients of thermal impedance in the Laplace domain in the form of ratio of polynomials. It is a crucial part of the implemented method. Finally, one can get thermal discrete time constant distribution (Foster network) and cumulative structure function (Cauer network) $[1,21,25]$.

\section{TOl methodology}

The new TOI methodology is presented in detail in fig. 1. It starts with pre-processing for data smoothing and noise reduction. In addition, for medical applications with IR temperature measurements, the movement correction of a patient is necessary [22]. Then, the Laplace transforms both for temperature and power are calculated. It leads directly to the thermal impedance in frequency domain $Z_{t h}(j \omega)$ typically represented in the Nyquist plot. The key step of the method is the approximation of thermal impedance in frequency domain by the ratio of polynomials. It is done by using the Least Square Method (LSM) [5-8]. Next, the Foster and Cauer network transformations are applied in order to support the physical interpretation of heat transfer in multilayer thermal structures. The very final stage of the proposed methodology is the convolution of the impulse function response (from Foster network) with a given power $p(t)$ to analyse the thermal response of the system for different excitations. It can be helpful e.g. in Non-Destructive Testing to estimate phase delay using a sinusoidal or periodic heating. 


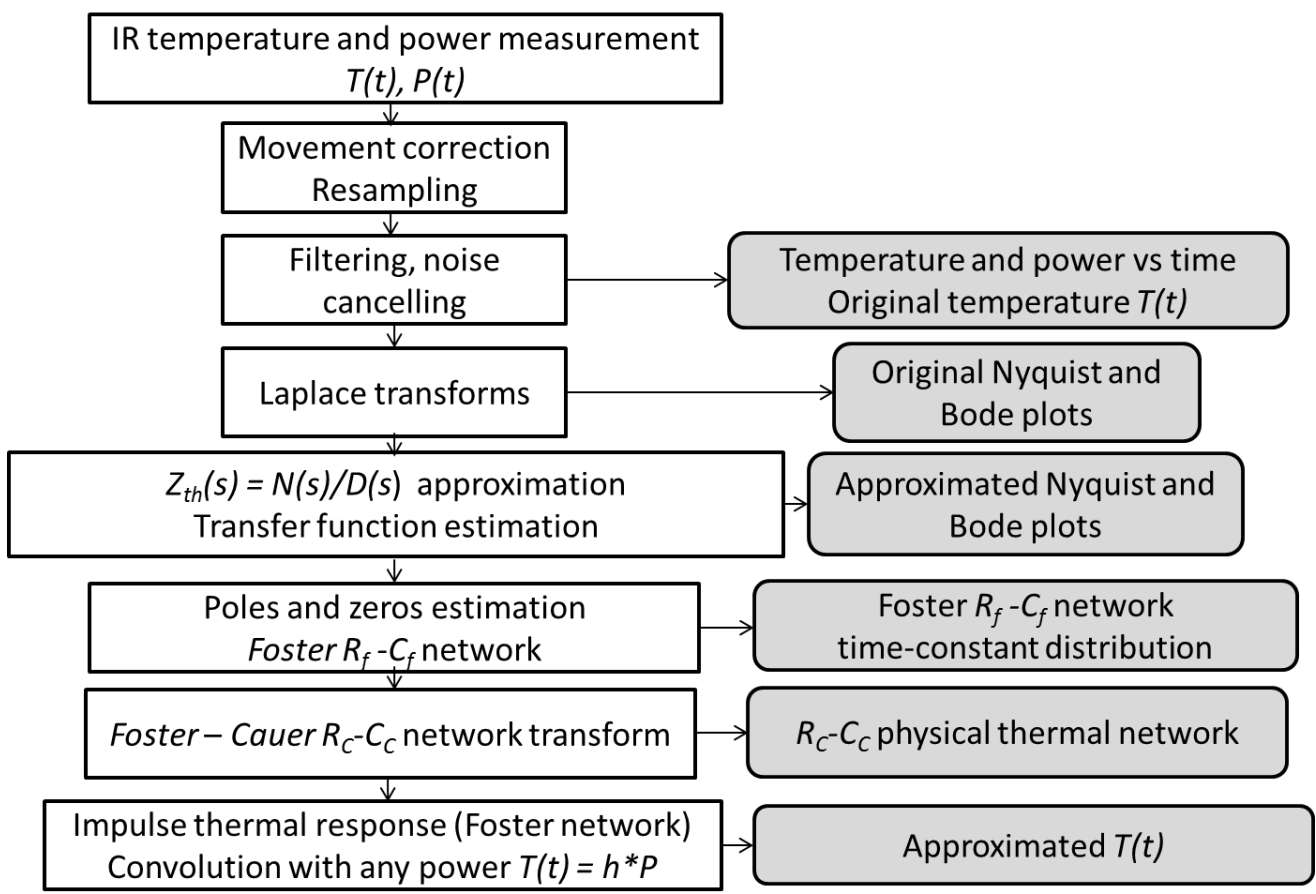

Fig. 1. TOI methodology in frequency domain

In order to calculate the complex thermal impedance $Z_{\text {th }}(j \omega)$, temperature and power measurements are needed. Thermal impedance in frequency domain is calculated by the Laplace transforms.

$$
T(s)=\int_{0}^{\infty} T(t) e^{-s t} d t, \quad P(s)=\int_{0}^{\infty} p(t) e^{-s t} d t
$$

where $\mathrm{s}=\mathrm{j} \omega, \omega$ is the angular frequency in $\mathrm{rad} / \mathrm{s}$.

Integrals in eq. (1) are numerically calculated using Filon or Simpson rules for even or uneven interval between the samples. One should bear in mind that Laplace transform needs integration up to infinity. Experimental data from any measurement stops after some time. It can cause the errors of the overall processing due to the finite time the temperature samples are available. The way of reducing this error is to wait relatively long time during measurements to reach almost steady state conditions and to approximate the last part of the signal by a constant value.

The result of the Laplace transformations gives the thermal impedance in the form $Z_{t h}(s)=\frac{T(s)}{P(s)}$, which can be graphically represented in the Nyquist plot for $s=j \omega$.

Next, the thermal impedance in frequency domain is approximated by a ratio of polynomials.

$$
Z_{t h}(s)=\frac{N(s)}{D(s)}
$$

According to the physical understanding of heat transfer in solids, the thermal system can be modelled by a lowpass transfer function. To fulfil the condition that the temperature vs. frequency in a monotonically decreasing function, the order denominator should always be greater at least by 1 than the order of the numerator. In other words, the number of zeros of the thermal systems is less by one than the number of poles, and they appear alternatively. The user can define the order of a thermal system taking into account, for example, the number of layers of the investigated structure. The general equation of the approximated thermal impedance function is presented in form of eq. (3).

where $n-$ is the order of thermal system.

$$
Z_{t h}(s)=\frac{\sum_{i=1}^{n} A_{i-1} s^{i-1}}{\sum_{i=1}^{n} B_{i} s^{i}+1}
$$

The approximation of the complex thermal impedance as a ratio of polynomials is calculated using LMS fitting algorithm for different frequency values (in practice for different angular frequencies $\omega_{k}, k=1,2 \ldots, N$ ). The number and distribution of these angular frequencies has to be selected carefully and they should cover the appropriate frequency range corresponding to the distribution of the thermal time constants of a thermal system. 
Because the result of the approximation depends on the distribution of frequencies the Nyquist plot is calculated for, the values of angular frequencies are chosen as it is presented in eq. (4).

$$
\omega_{k}=\omega_{1} e^{\alpha(k-1)}
$$

where $k=1, \ldots N$ is the number of angular frequency, and $\omega_{1}$ is the lowest value, the Nyquist plot and complex thermal impedance are calculated for.

If one assumes to analyse thermal impedance in the range $\omega \in\left[\omega_{1}, \omega_{N}\right]$, the distribution coefficient is equal to $\alpha=\left(\ln \omega_{N}-\ln \omega_{1}\right) / N$. Frequencies $\omega_{1}, \omega_{N}$ should be lower and greater than $\omega_{1}<1 / \tau_{1}$ and $\omega_{N}>1 / \tau_{N}$ by at least one octave, where $\tau_{1}$ and $\tau_{N}$ are the largest and the smallest thermal time constants of an identified object.

The important issue of the overall algorithm is the low-pass filtering of input (power) and output (temperature) signals before LMS fitting. There are many scientific discussions on this topic [5-9]. Such filtering significantly reduces the noise of the signals and makes whole algorithm more stable and precise. There are different filters proposed in the literature. One of the possible filters is given in eq. (5)

$$
F(s)=\frac{k_{f}}{\left(1+s \tau_{f}\right)^{n_{f}}}
$$

where the order of the filter $n_{f}$ should be comparable with the order of a thermal system.

We notice, that in general case $n_{f}$ can take either an integer or a real value. Taking into account eq. (3), the optimisation problem of finding the unknown values of the coefficients $A_{i}$ and $B_{i}$ consists in minimizing the function (6).

$$
\sum_{s=j \omega_{1}}^{s=j \omega_{N}}\left[X(s) F_{j}(s) \sum_{i=1}^{n} A_{i-1} s^{i-1}-Y(s) F_{j}(s)\left(\sum_{i=1}^{n} B_{i} s^{i}+1\right)\right]^{2}=\min
$$

One has to underline, that in general case the filter can iteratively vary during optimisation to get the better fitting. It leads to a nonlinear LSM approximation discussed in the literature $[5-9,26]$.

It is convenient to rewrite eq. (3) of thermal impedance in the multiplicative form (7).

$$
Z_{t h}(s)=\frac{A_{n-1}}{B_{n}} \frac{\left(s+s_{z n-1}\right) \ldots\left(s+s_{z 1}\right)}{\left(s+s_{p n}\right)\left(s+s_{p n-1}\right) \ldots\left(s+s_{p 1}\right)}
$$

The roots of denominator of eq. (7) correspond to the thermal time constants of the Foster $R_{f}-C_{f}$ network.

$$
D(s)=B_{n} s^{n}+B_{n-1} s^{n-1}+\cdots+B_{1} s+1=0
$$

In typical TOI process, the poles of the thermal impedance $Z_{t h}(j \omega)$ should be real and negative. It happens if the original thermal impedance obtained from eq. (1) is correct, the temperature and power are the smooth curves without much of noise, and the thermal process reaches the steady state. Poles of $Z_{\text {th }}(j \omega)$ i.e. the roots of denominator $D(j \omega)$ denote the angular frequencies of Foster $R_{f}-C_{f}$ network and can be expressed as:

$$
\omega_{i}=\frac{1}{\tau_{i}}=\frac{1}{R_{f i} C_{f i}}=-s_{p i}
$$

In this way, one can get the Foster thermal $R_{f}-C_{f}$ network - eq. (10).

$$
Z_{t h}(s)=\sum_{i=1}^{n} \frac{k_{i}}{s+s_{p i}}=\sum_{i=1}^{n} \frac{R_{f i}}{s \tau_{f i}+1}
$$

where $R_{f i}$ can be easily calculated using the residues of thermal impedance (3).

$$
R_{f i}=\underset{s=s_{p i}}{\operatorname{res}}\left[\frac{N(s)}{D(s)}\right]=\lim _{s \rightarrow s_{p i}}\left[\frac{N(s)\left(s-s_{p i}\right)}{D(s)}\right]=\frac{N(s)}{D^{\prime}(s)}
$$

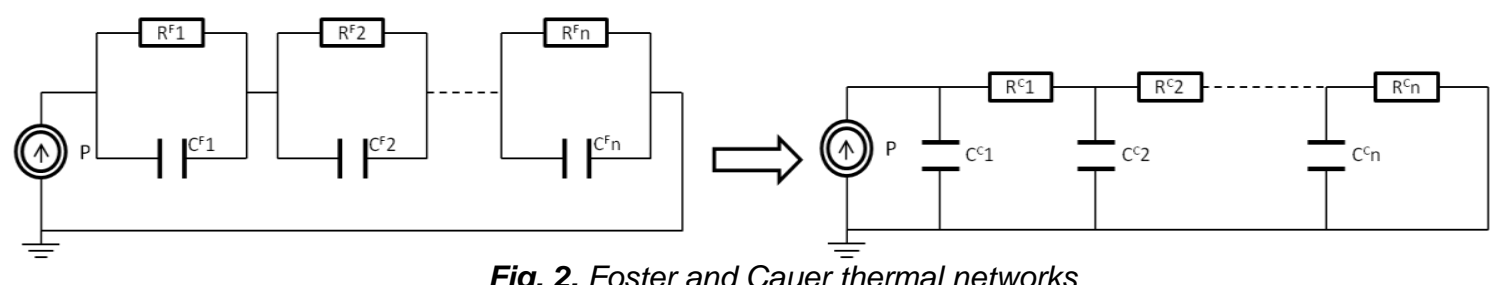

Fig. 2. Foster and Cauer $\overline{\bar{t}}$ hermal networks

Next step of the TOI methodology is the transformation of the Foster to the equivalent Cauer network (fig. 2). The thermal resistances and capacitances $R_{C i}$ and $C_{C i}$ are calculated by multiple polynomial divisions [17]. 


$$
Z_{t h}(s)=\frac{1}{s C_{C 1}+\frac{1}{R_{C 1}+\frac{1}{s C_{C 2}+\frac{1}{R_{C 2}+\cdots}}}}
$$

From the Foster representation (10), the impulse thermal response of the thermal system in time domain can be presented in the form of eq. (13).

$$
h_{t h}(t)=\sum_{i=1}^{n} R_{i} e^{-\frac{t}{\tau_{i}}}
$$

Finally, by performing a convolution of the thermal impulse response in time domain with any given power, one can calculate the thermal response of structure for different excitations.

where * is convolution.

$$
\tilde{T}(t)=\tilde{P}(t) * h_{t h}(t)=\tilde{P}(t) * \sum_{i=1}^{n} R_{i} e^{-\frac{t}{\tau_{i}}}
$$
analysis.

Comparison of temperature measurement $T(t)$ and the result of convolution $\tilde{T}(t)$ confirms the correctness of the

\section{TOI software}

The TOI program is written in MATLAB environment. The input data can be loaded from the file as the functions of time. The power can be defined or loaded by the users together with the temperature input data. The program allows calculating:

- $\quad$ thermal impedance in frequency domain $Z_{t h}(j \omega)$ smoothed by approximation using ratio of polynomials representation of the thermal impedance in a Nyquist plot,

- magnitude and phase characteristics, $\operatorname{modulus}\left\{Z_{t h}(j \omega)\right\}$, angle $\left\{Z_{t h}(j \omega)\right\}-$ Bode plots,

- discrete thermal time constants distribution $R(\tau)$ - Foster network,

- cumulative structure function - Cauer network,

- temperature response vs. time for a given power.

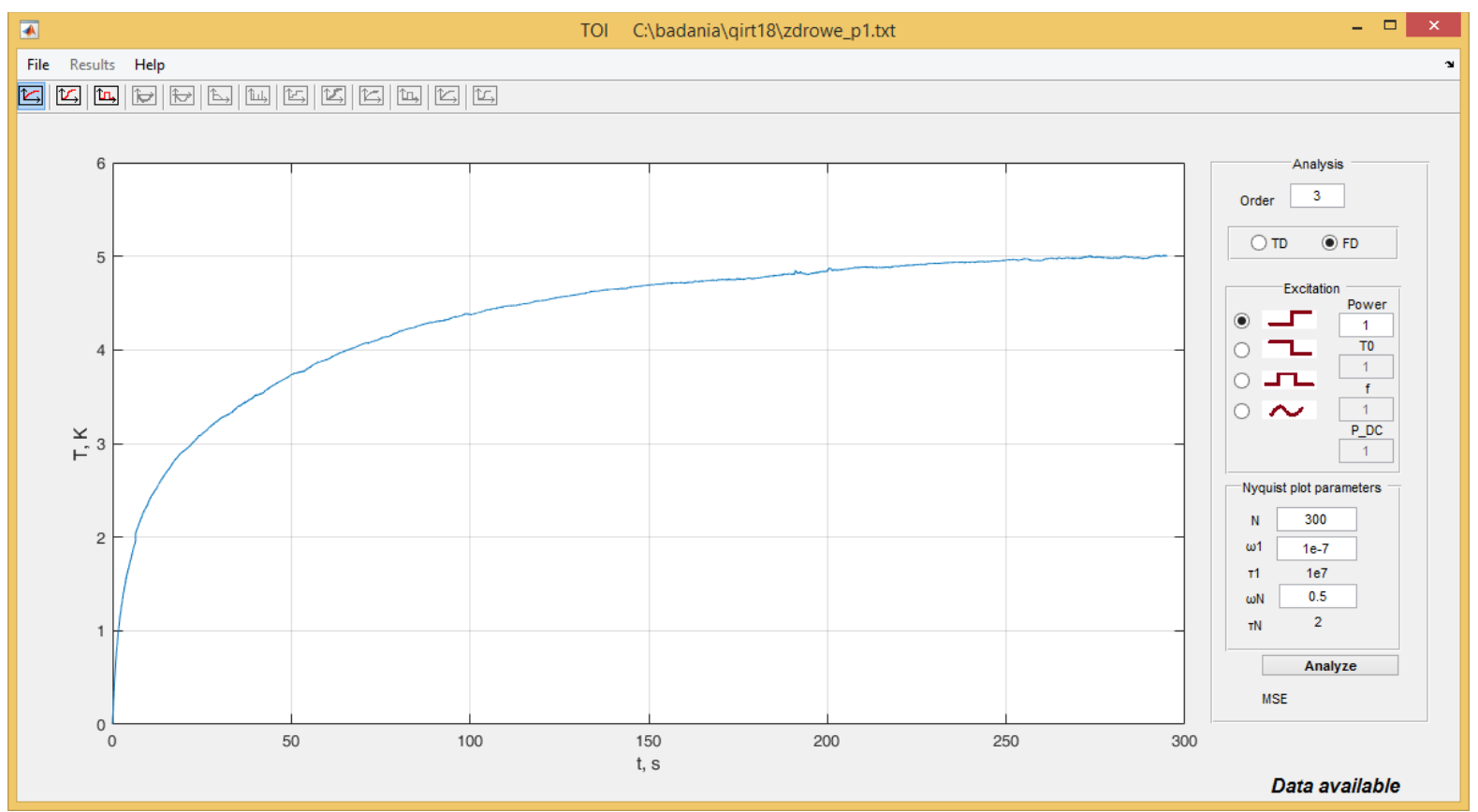

Fig. 3. Main screen of TOI program

The TOI program is intuitive and user-friendly. The functions are simple activated by buttons with graphical icons. The results are presented either graphically by plotting curves or numerically in the form of tables. All calculated data like approximated Nyquist plot for specified frequencies, coefficients of polynomials, values of $R_{i}$ and $C_{i}$ for Foster and Cauer networks can be saved in the Excel file for further use. In addition, all plots generated by the program can be 
stored on the disk as well. It can be helpful to create final reports from the analysis. For comparative reasons, it is possible to perform analysis either in frequency $(F D)$ or time domain $(T D)$, as it is shown in fig. 1 . The program is available with the Freeware licence on the website of the first co-author from the Lodz University of Technology.

\section{Exemplary results}

In order to verify the TOI methodology, we tested the program for a given complex thermal impedance. A semiinfinite solid material with a small heat source on top was chosen [1]. It can be the model of a small microelectronic device on the surface of a thick semiconductor substrate. The thermal impedance of this thermal system can be presented in form of eq. (15).

$$
Z_{t h}(s)=\frac{Z_{t h 0}}{1+\sqrt{s \tau_{0}}}
$$

It can be proven that the time constant distribution $R_{t h}(\tau)$ is a continuous function with the maximum value for $\tau=$ $\tau_{0}$. Function $R_{t h}(\tau)$ can be analytically derived and it takes form (16) [1].

$$
R_{t h}(\tau)=\frac{Z_{t h 0}}{\pi} \frac{\sqrt{\frac{\tau}{\tau_{0}}}}{1+\frac{\tau}{\tau_{0}}}
$$

Continuous time constant distribution means infinite number of discrete time constants. From practical point of view, the number of time constants can always be limited. In this example, in order to get the reference results, we used the in-built function available in MATLAB program for dynamic system identification (Transfer Function Estimation - tfest) [26]. The results for $Z_{\text {tho }}=5 \mathrm{~K} / \mathrm{W}$ and $\tau_{0}=75 \mathrm{~s}$, and for $n=15$ time constants are presented in figs. 4-6.

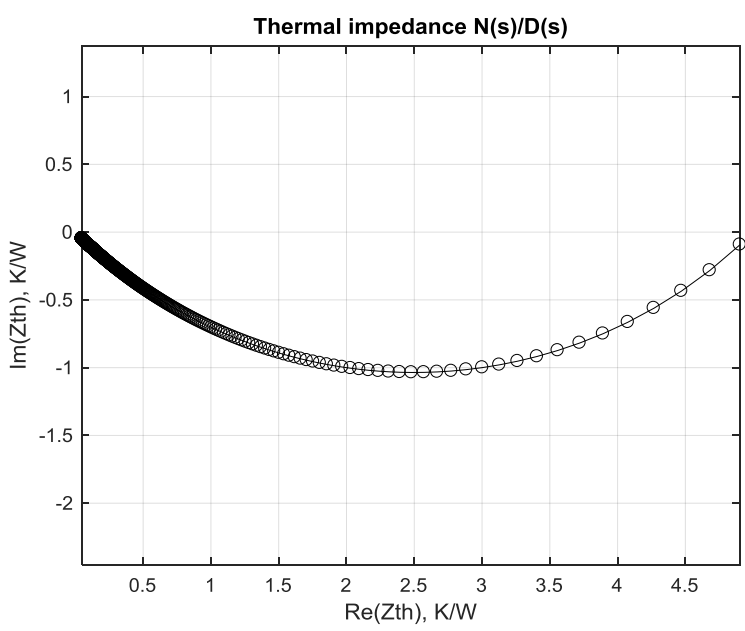

Fig. 4. The Nyquist plot of the complex thermal impedance defined by eq. (15)

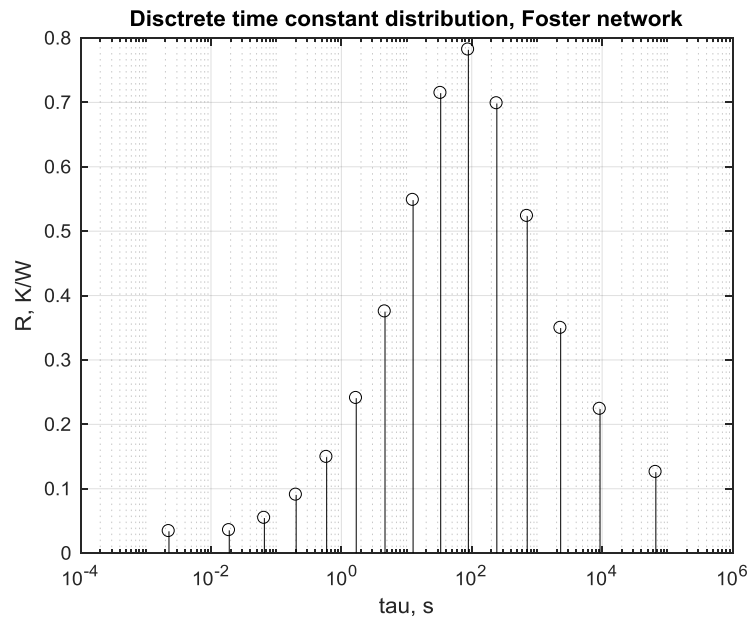

Fig. 5. Discrete time constant distribution $(n=15)$ for the thermal impedance (15)

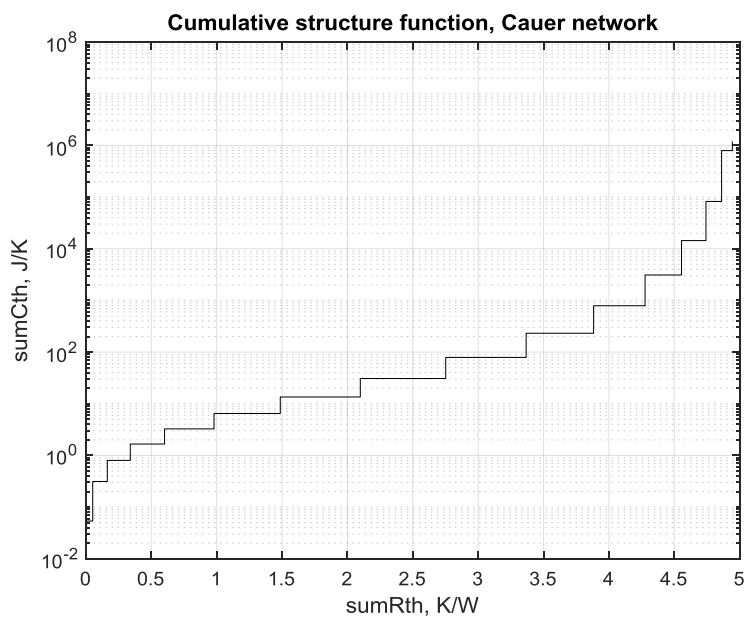

Fig. 6. Cumulative structure function for the thermal impedance (15) 
The presented software is intended for use in both technical and biomedical applications. We performed a medical experiment to investigate the thermal parameters for a skin with and without visible inflammation. The skin was cooled down by a few degrees using special cooling devices [22]. We tested gels, metal blocks pre-cooled in a fridge or an electronic cooler equipped with Peltier elements. After few seconds of cooling, the cooling device was removed and temperature rise was registered by the fast cooled photon thermal camera. The results of thermal analysis using TOI concept are shown below in figs 7-11.

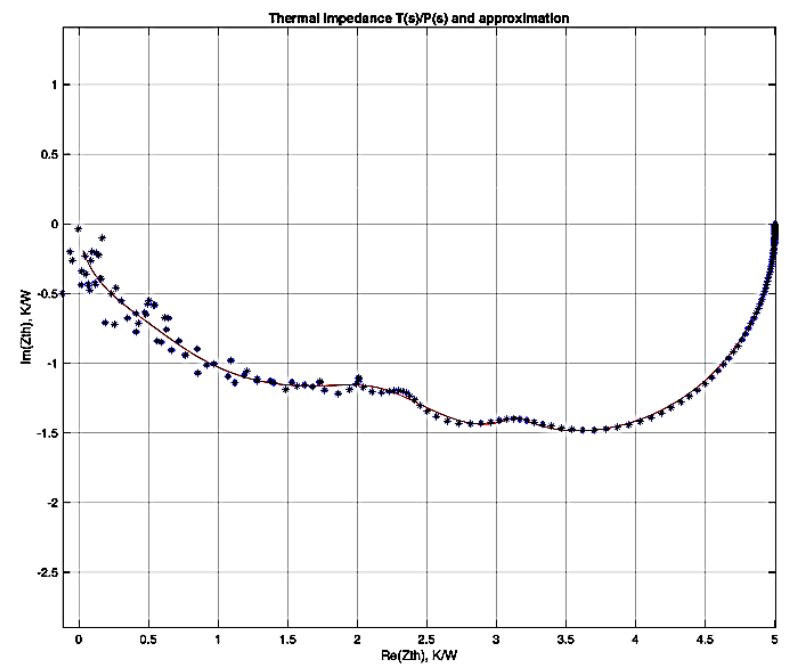

Fig. 7. Original and approximated complex thermal impedance $Z_{t h}(j \omega)-$ Nyquist plots

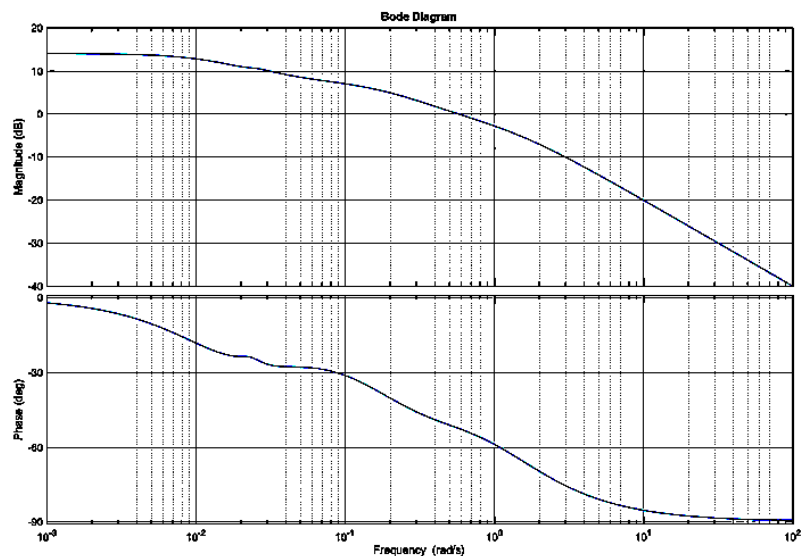

Fig. 8. Magnitude and phase for approximated complex thermal impedance $Z_{\text {th }}(j \omega)$ - Bode plots

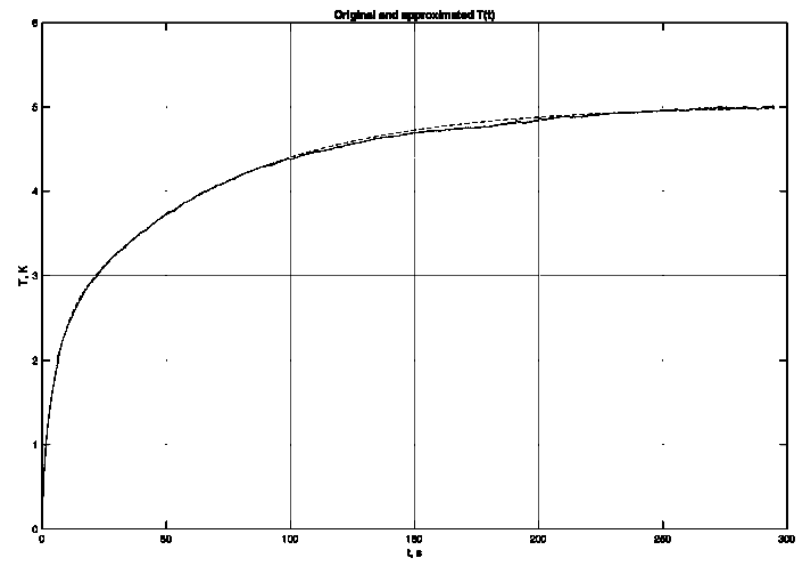

Fig. 9. Comparison of step function response for thermographic measurement and approximated $Z_{\text {th }}(j \omega)=$ $N(j \omega) / D(j \omega)$ (after convolution) 


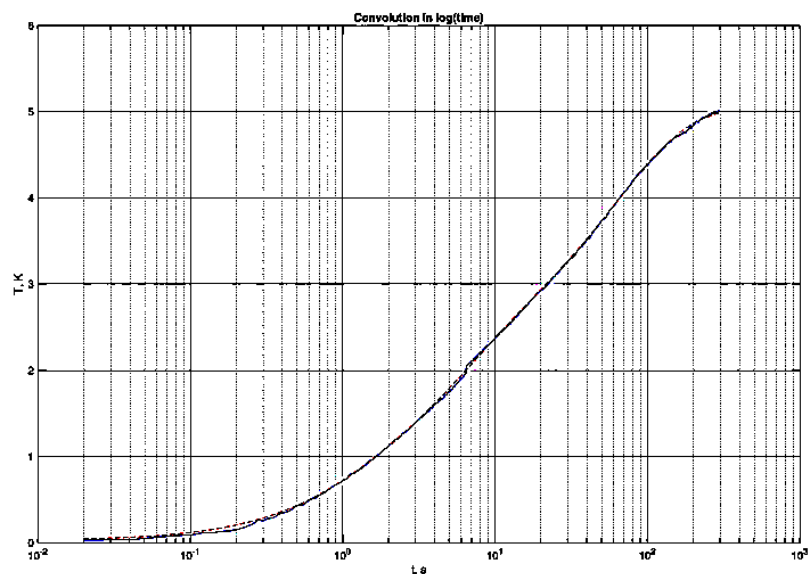

Fig. 10. Comparison of temperature step function response in log scale for thermographic measurement and approximated $Z_{\text {th }}(j \omega)=N(j \omega) / D(j \omega)$ (after convolution)

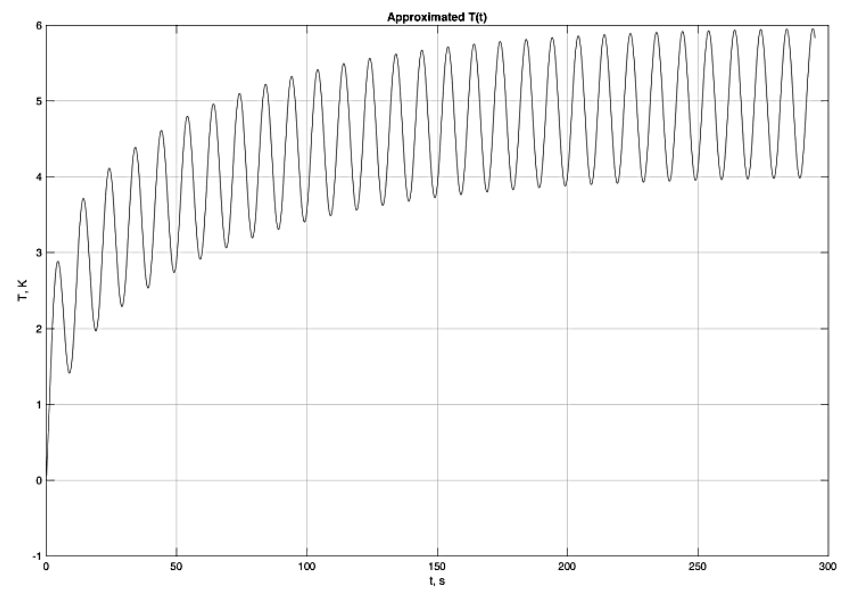

Fig. 11. Exemplary temperature response of the system for sinusoidal excitation

\section{Conclusions}

The new software for Thermal Object Identification in frequency domain was presented. It can be effectively used with contactless measurements performed using thermal cameras. The software allows approximating the thermal system either by the Foster network - discrete time constant distribution, or Cauer one - the cumulative structure function. Obviously, the faster the IR thermographic camera, the shorter time constants can be identified. The reports are generated by the software in graphical and numerical forms. The user can verify the correctness of the result by comparing measured temperature with the approximated one using TOI methodology both in linear and logarithmic scales. The software is available under the Freeware licence regulation. It is planned to link TOI software to any IR camera having the drivers both for Windows and Linux systems (possible to Android as well).

\section{REFERENCES}

[1] Vermeersch B.: Thermal AC modelling, simulation and experimental analysis of microelectronic structures including nanoscale and high-speed effects. Ph.D. Thesis, Gent University, ELIS, Belgium, 2009.

[2] Szekely V.: On the representation of infinite-length distributed RC oneports. IEEE Trans. Circuits Syst., vol. 38, pp. 711-719, 1991.

[3] Szekely V.: Identification of RC networks by deconvolution: Chances and limits. IEEE Trans. Circuits Syst., vol. 45, no. 3, pp. 244-258, 1998.

[4] Marco S., Palcin J. Samitier J., Improved multiexponential transient spectroscopy by iterative deconvolution, IEEE Trans. In Instrumentation and Measurement, vol. 50, pp. 774-780, 2001.

[5] Garnier, H., Mensler M., Richard A., "Continuous-time Model Identification From Sampled Data: Implementation Issues and Performance Evaluation." International Journal of Control, 2003, Vol. 76, Issue 13, pp. 1337-1357.

[6] Ljung, L. "Experiments With Identification of Continuous-Time Models." Proceedings of the 15th IFAC Symposium on System Identification. 2009. 
[7] Young, P. C. and A.J. Jakeman. Refined instrumental variable methods of time-series analysis: Part III, extensions. International Journal of Control 31, 1980, pp 741-764.

[8] Drmac, Z., Gugercin S., Beattie C., Quadrature-based vector fitting for discretized H2 approximation. SIAM Journal on Scientific Computing. Vol. 37, Numer 2, 2014, pp A625-A652.

[9] Ozdemir A., Gumussoy S., "Transfer Function Estimation in System Identification Toolbox via Vector Fitting." Proceedings of the 20th World Congress of the International Federation of Automatic Control. Toulouse, France, July 2017.

[10] Górecki K., Zarębski J.: The influence of the selected factors on transient thermal impedance of semiconductor devices. Proceedings of the 21st International Conference Mixed Design of Integrated Circuits and Systems MIXDES, Lublin, pp. 309-314, 2014.

[11] Górecki K., Rogalska M., Zarębski J.: Parameter estimation of the electrothermal model of the ferromagnetic core. Microelectronics Reliability, Vol. 54, No. 5, pp. 978-984, 2014.

[12] Russo S.: Measurement and simulation of electrothermal effects in solid-state devices for RF applications. 2010.

[13] Protonotarios E.N., Wing O.: Theory of Nonuniform RC Lines, Part I: Analytic Properties and Realizability Conditions in the Frequency Domain. IEEE Transactions on Circuit Theory, vol. 14, no. 1, pp. 2-12, 1967.

[14] Büttner W.: Ein numerisches Verfahren zur Exponential approximation von transienten Wärmewiderständen. Archiv for Elektrotechnik, vol. 59, no. 6, pp. 351-359, 1977.

[15] Jibia A.U., Salami M-J: An Appraisal of Gardner Transform-Based Method of Transient Multiexponential Signal Analysis. International Journal of Computer theory and Engineering, vol.4, pp. 16-24, 2012.

[16] Hellen E. H.: Pade-Laplace analysis of signal averaged voltage decays obtained from a simple circuit. American Journal of Physics, vol. 73, no.9, Sept. 2005, pp. 871-875.

[17] Murthy K., Bedford R.: Transformation between Foster and Cauer equivalent networks. IEEE Transactions on Circuits and Systems, vol. 25, no. 4, pp. 238-239, 1978.

[18] Chatzipanagiotou P., Chatziathanasiou V., De Mey G., Wiecek B.: Influence of soil humidity on the thermal impedance, time constant and structure function of underground cables: A laboratory experiment. Applied Thermal Engineering, vol. 113, pp. 1444-1451, 2017.

[19] Chatziathanasiou V., Chatzipanagiotou P., Papagiannopoulos I., De Mey G., Wiecek B.: Dynamic thermal analysis of underground medium power cables using thermal impedance, time constant distribution and structure function. Applied Thermal Engineering, vol. 60, no. 1-2, pp. 256-260, 2013.

[20] Kałuża M., Więcek B., De Mey G., Hatzopoulos A., Chatziathanasiou V.: Thermal impedance measurement of integrated inductors on bulk silicon substrate. Microelectronics Reliability, vol. 73, pp. 54-59, 2017.

[21] Chatzipanagiotou P., Strąkowska M., De Mey G., Chatziathanasiou V., Więcek B., A new software tool for transient thermal analysis based on fast IR camera temperature measurement, Measurement Automation Monitoring, Feb. 2017, no. 62, vol. 63, ISSN 2450-2855, pp. 49-51.

[22] Strakowska M., Strąkowski R., Strzelecki M., De Mey G., Wiecek B., Thermal modelling and screening method for skin pathologies using active thermography, Biocybernetics and Biomedical Engineering, 10.1016/j.bbe.2018.03.009.

[23] Jakopovid Z., Bencic Z., Koncar R.: Identification of Thermal Equivalent - Circuit Parameters for Semiconductors. IEEE Workshop on Computers in Power Electronics, pp. 251-260, 1990.

[24] Savitzky A., Golay M.J.E.: Smoothing and differentiation of data by simplified least squares procedures. Anal. Chem., vol. 36, pp. 1627-1639, 1964.

[25] T3Ster-Master Thermal Evaluation Tool - User's Manual Version 2.2, Mentor Graphics Corporation.

[26] https://nl.mathworks.com/help/ident/ref/tfest.html 